\title{
Existence and uniqueness of solutions of stochastic functional differential equations
}

\author{
Max-K. von Renesse and Michael Scheutzow
}

Communicated by S. Molchanov

\begin{abstract}
Using a variant of the Euler-Maruyama scheme for stochastic functional differential equations with bounded memory driven by Brownian motion we show that only weak one-sided local Lipschitz (or "monotonicity") conditions are sufficient for local existence and uniqueness of strong solutions. In case of explosion the method yields the maximal solution up to the explosion time. We also provide a weak growth condition which prevents explosions to occur. In an appendix we formulate and prove four lemmas which may be of independent interest: three of them can be viewed as rather general stochastic versions of Gronwall's Lemma, the final one provides tail bounds for Hölder norms of stochastic integrals.
\end{abstract}

Keywords. Stochastic functional differential equation, existence of solution, maximal solution, uniqueness of solution, Dereich lemma, stochastic Gronwall lemma.

2000 Mathematics Subject Classification. 34K50, 34K05, 60H10, 60H20.

\section{Introduction}

There is by now a rather comprehensive mathematical literature on the mathematical theory and on applications of stochastic functional (or delay) differential equations driven by Brownian motion. Existence and uniqueness of global solutions have been established under global Lipschitz conditions on the coefficients (e.g. [10]) or under local Lipschitz and linear growth conditions (e.g. [9, 12]). On the other hand it is common knowledge for non-delay (stochastic) differential equations that only one-sided Lipschitz conditions are sufficient for local existence of solutions. This distinction becomes particularly relevant in infinite dimensions where the drift in (stochastic) evolution equations is unbounded and discontinuous in almost all interesting cases but nevertheless satisfies a one-sided Lipschitz. i.e. "monotonicity/dissipativity" condition, cf. e.g. [11]. In this paper we show that monotonicity of the coefficients guarantees local existence of solutions to delay equations with bounded memory, thereby closing a systematic gap in the existing literature. 
We choose the classical framework of the space of continuous functions as a natural state space of the equation. Note that, due to the absence of an inner product on this space, the right formulation of monotonicity is not obvious in this case. The proposed condition (M) below fits well to our needs, since it recovers the classical monotonicity condition for the non-delay case as a limit and yet is weak enough to cover a rather big set of equations.

In our proof we define a specific Euler-Maruyama scheme, which is generally a very powerful tool in the Markovian case $[1,6,7]$. Other variants have been treated for the numerical simulation of stochastic delay equations under Lipschitz conditions in e.g. [4, 5, 8] and most recently [3]. We point out that our method yields an approximation in the strong sense even in the case of an explosion. In particular our proof below shows how the explosion time can be recovered numerically, which seems to be a question typically neglected in the literature.

As for the proofs, note that the left hand side of condition (M) is quite weak w.r.t. the $C^{0}$-norm. As a consequence the standard two-step Burkholder-DavisGundy and Gronwall argument cannot be applied to obtain the crucial contraction estimates. We overcome this difficulty by what we call stochastic Gronwall lemmas and which are presented in the appendix. We think that they may be of independent interest. These lemmas are also crucial for the global existence assertion which holds under a rather familiar growth (or "coercivity", [11]) condition (C), which is again weak in the $C^{0}$-topology.

\section{Set up and main results}

For $r>0$, let $\zeta$ denote the space of continuous $\mathbb{R}^{d}$-valued functions on $[-r, 0]$ endowed with the sup-norm $\|\cdot\|$. For a function or a process $X$ defined on $[t-r, t]$ we write $X_{t}(s):=X(t+s), s \in[-r, 0]$. Consider the stochastic functional differential equation

$$
\left\{\begin{aligned}
\mathrm{d} X(t) & =f\left(X_{t}\right) \mathrm{d} t+g\left(X_{t}\right) \mathrm{d} W(t), \\
X_{0} & =\varphi,
\end{aligned}\right.
$$

where $W$ is an $\mathbb{R}^{m}$-valued Brownian motion defined on a complete probability space $(\Omega, \mathcal{F}, \mathbb{P})$ with the augmented Brownian filtration

$$
\mathscr{F}_{t}{ }^{W}=\sigma(W(u), 0 \leq u \leq t) \vee \mathcal{N} \subset \mathcal{F},
$$

where $\mathcal{N}$ denotes the null-sets in $\mathcal{F}, \varphi$ is an $\left(\mathcal{F}_{t}{ }_{t}\right)$-independent $\mathcal{C}$-valued random variable and $f: \mathcal{C} \rightarrow \mathbb{R}^{d}, g: \mathcal{C} \rightarrow \mathbb{R}^{d \times m}$ are continuous maps. 
We will suppose throughout this work the following monotonicity assumption on $f$ and $g$ :

For each compact subset $C \subset \mathcal{C}$, there exists a number $K_{C}$ and some $r_{C} \in$ $(0, r]$ such that for all $x, y \in C$ with $x(s)=y(s) \forall s \in\left[-r,-r_{C}\right]$

$$
2\langle f(x)-f(y), x(0)-y(0)\rangle+\|\| g(x)-g(y)\left\|^{2} \leq K_{C}\right\| x-y \|^{2},
$$

where $\langle\cdot, \cdot\rangle$ denotes the standard inner product on $\mathbb{R}^{d}$ and $\|M \mid\|^{2}=\operatorname{tr}\left(M M^{*}\right)$ for $M \in \mathbb{R}^{d \times m}$.

As an example in $d=1$ take $f(x)=\varphi\left(\sum_{i=1}^{N} w_{i} x\left(t_{i}\right)\right)$, where $t_{i} \in[-r, 0]$, $w_{i} \geq 0, i=1, \ldots, N$, and $\varphi \in C(\mathbb{R})$ is a non-increasing continuous (not necessarily Lipschitz) function, e.g. $\varphi(s)=-\operatorname{sign}(s) \sqrt{|s|}$ and $g$ locally Lipschitz on $\mathcal{C}$. Another example is $f=f_{1}+f_{2}+f_{3}$ with $f_{1}$ locally Lipschitz on $\mathcal{C}, f_{2}(x)=\int_{-r}^{-r_{0}} \psi(x(s)) k(s) \mathrm{d} s$ for some $0<r_{0}<r, k, \psi \in C(\mathbb{R})$ and $f_{3}(x)=\varphi(x(0))$ with $\varphi \in C(\mathbb{R})$ non-increasing as above.

Our first result is a local existence and uniqueness statement for solutions to (2.1) for which we recall some basic notions. Given any filtration $\left(\mathcal{F}_{t}\right)$ on $\Omega$, an $\left(\mathcal{F}_{t}\right)$-stopping time $\sigma: \Omega \rightarrow \overline{\mathbb{R}}_{\geq 0}$ is called predictable if there exists a sequence of ("announcing") stopping times $\sigma_{n}$ such that $\sigma_{n}<\sigma$ and $\sigma_{n} \nearrow \sigma \mathbb{P}$-almost surely.

A tuple $X=(X, \sigma)$ of a predictable stopping time $\sigma$ and a map $X: \Omega \times([-r, 0] \cup$ $[0, \sigma)) \rightarrow \mathbb{R}^{d}$ is called a local $\left(\widetilde{F}_{t}\right)$-semimartingale up to time $\sigma$ starting from $\varphi \in \mathcal{C}$, if $X_{0}=\varphi$ holds $\mathbb{P}$-almost surely and for any (announcing) stopping time $\sigma_{n}<\sigma$, the process $\left(X^{\sigma_{n}}(t)\right)_{t \geq 0}$ with $X^{\sigma_{n}}(t)=X\left(t \wedge \sigma_{n}\right)$ is an $\mathbb{R}^{d}$-valued $\left(\mathcal{F}_{t}\right)$-adapted semimartingale.

Definition 2.1 (Local Solution). Let $\mathscr{F}_{t}=\mathscr{F}_{t}{ }^{W} \vee \sigma(\varphi)$. A local $\left(\mathscr{F}_{t}\right)$-semimartingale $(X, \sigma)$ up to a predictable stopping time $\sigma$ is called a local strong solution to equation (2.1) if $X_{0}=\varphi$ and for any stopping time $\sigma_{n}<\sigma$ and any $t \geq 0$

$$
X\left(t \wedge \sigma_{n}\right)=X(0)+\int_{0}^{t \wedge \sigma_{n}} f\left(X_{u}\right) \mathrm{d} u+\int_{0}^{t \wedge \sigma_{n}} g\left(X_{u}\right) \mathrm{d} W(u) \quad \mathbb{P} \text {-a.s. }
$$

The pair $(X, \sigma)$ is called maximal strong solution if in addition $\left(X_{t}\right)$ eventually leaves any compact set $K \subset \mathcal{\ell}$ for $t \rightarrow \sigma, \mathbb{P}$-almost surely on $\{\sigma<\infty\}$; i.e.

$$
\mathbb{P}\left(\left\{\exists \text { a compact set } K \subset \mathcal{C} \text { and } t_{i} \nearrow \sigma \text { s.t. } X_{t_{i}} \in K\right\} \cap\{\sigma<\infty\}\right)=0 .
$$

Theorem 2.2. Equation (2.1) admits a unique maximal strong solution $(X, \sigma)$ provided (M) holds. 
Theorem 2.3. In addition to the assumptions of Theorem 2.2 let $f$ and $g$ be bounded on bounded subsets of $\mathcal{C}$ and let the pair $(f, g)$ be weakly coercive in the sense that there exists a non-decreasing function $\rho:[0, \infty) \rightarrow(0, \infty)$ such that $\int_{0}^{\infty} 1 / \rho(u) \mathrm{d} u=\infty$ and for all $x \in \mathcal{C}$

$$
2\langle f(x), x(0)\rangle+\||| g(x) \mid\|^{2} \leq \rho\left(\|x\|^{2}\right) .
$$

Then $X$ is globally defined, i.e. $\sigma=\infty \mathbb{P}$-almost surely.

\section{Proof of Theorem 2.2}

The proof of Theorem 2.2 is based on an iteration of Lemma 3.1 below, which requires some auxiliary notation. For $\Phi \subset \mathcal{C}$ and $R>0$ let

$$
\begin{array}{r}
C_{\Phi, R}=\left\{\eta \in \mathcal{C} \mid \exists \varphi \in \Phi, r_{0} \in[0, r]: \eta(u)=\varphi\left(u+r_{0}\right), u \in\left(-r,-r_{0}\right],\right. \\
\left.\|\eta-\varphi(0)\|_{1 / 4 ;\left[-r_{0}, 0\right]} \leq R\right\} \subset \mathcal{C},
\end{array}
$$

where

$$
\|\eta\|_{\alpha ;[a, b]}=\sup _{a \leq u<v \leq b}\left(|\eta(v)-\eta(u)| /(v-u)^{\alpha}\right)+\sup _{a \leq u \leq b}|\eta(u)|
$$

denotes the Hölder- $\alpha$-norm on $C\left([a, b], \mathbb{R}^{d}\right), \alpha \in(0,1)$.

Note that $C_{\Phi, R}$ is compact in $\mathcal{C}$ provided $\Phi$ is.

Below we drop the subscript $\Phi$ whenever this causes no confusion.

Lemma 3.1. In addition to the conditions of Theorem 2.2 assume there is a compact subset $\Phi \subset \mathcal{C}$ such that $\varphi \in \Phi \mathbb{P}$-almost surely. For $R>0$, let $r_{R}=r_{C}$ be the constant appearing in (M) for choosing $C=C_{\Phi, R}$. Then there exists a stopping time $0<\sigma_{R} \leq r_{R}$ and a unique (up to indistinguishability) ( $\mathcal{F}_{t}$ )-adapted process $X(t), t \in\left[0, \sigma_{R}\right]$ such that $X_{t} \in C_{R}$ for all $t \in\left[0, \sigma_{R}\right]$ which solves (2.1) up to time $\sigma_{R}$. Moreover,

$$
\|X(\cdot)-\varphi(0)\|_{1 / 4 ;\left[0, \sigma_{R}\right]} \geq \frac{R}{2} \quad \mathbb{P} \text {-a.s. on }\left\{\sigma_{R}<r_{R}\right\} .
$$

Proof. The proof is inspired by the arguments for finite dimensional monotone SDEs in [7], cf. e.g. [11]. For $n \in \mathbb{N}$, we define an Euler-like approximation to (2.1) with step size $\frac{1}{n}$ by

$$
\left\{\begin{aligned}
\mathrm{d} X^{n}(t) & =f\left(\bar{X}_{t}^{n}\right) \mathrm{d} t+g\left(\bar{X}_{t}^{n}\right) \mathrm{d} W(t), \\
X_{0}^{n} & =\varphi
\end{aligned}\right.
$$


where we define $\bar{X}_{s}^{n}(\cdot) \in \mathcal{C}, s \geq 0$, by

$$
\bar{X}_{s}^{n}(u)=X^{n}\left((s+u) \wedge \frac{\lfloor n s\rfloor}{n}\right), \quad u \in[-r, 0] .
$$

Equation (3.2) admits a global in time solution via the recursion $X_{0}^{n}=\varphi$ and

$$
X^{n}(t)=X^{n}\left(\frac{\lfloor n t\rfloor}{n}\right)+\int_{\lfloor n t\rfloor / n}^{t} f\left(\bar{X}_{s}^{n}\right) \mathrm{d} s+\int_{\lfloor n t\rfloor / n}^{t} g\left(\bar{X}_{s}^{n}\right) \mathrm{d} W(s) .
$$

The process $t \mapsto X^{n}(t)$ is adapted and continuous, hence

$$
t \mapsto p_{t}^{n}(\cdot):=\bar{X}_{t}^{n}(\cdot)-X_{t}^{n}(\cdot), t \geq 0,
$$

defines an adapted $\mathcal{C}$-valued process (which is càdlàg). With this, (3.2) is equivalent to $X_{0}^{n}=\varphi$ and

$$
X^{n}(t)=\varphi(0)+\int_{0}^{t} f\left(X_{s}^{n}+p_{s}^{n}\right) \mathrm{d} s+\int_{0}^{t} g\left(X_{s}^{n}+p_{s}^{n}\right) \mathrm{d} W(s) .
$$

Without loss of generality, we may assume that the set $\Phi$ has the property that $0 \in \Phi$ and $\eta \in \Phi, s \in[-r, 0)$ implies that the function $u \mapsto \eta(u \wedge s), u \in[-r, 0]$ also belongs to $\Phi$. Then, $X_{t}^{n} \in C_{R}$ implies $\bar{X}_{t}^{n} \in C_{R}$, hence $p_{t}^{n} \in \widetilde{C}_{R}=$ $\left\{\eta_{1}-\eta_{2} \mid \eta_{i} \in C_{R}\right\}$ provided

$$
t \leq \tau_{R}^{n}:=\inf \left\{t>0 \mid X_{t}^{n} \notin C_{R}\right\} .
$$

Since $\widetilde{C}_{R} \subset \mathcal{C}$ is again compact,

$$
\widetilde{\rho}(R)=\sup _{x \in \widetilde{C}_{R}}\|x\|<\infty
$$

and the continuity of $f$ and $g$ ensures that

$$
C_{1}(R):=\sup _{x \in \widetilde{C}_{R}}\{|f(x)|+\||g(x) \||\}<\infty .
$$

Fix $n, m \in \mathbb{N}$ and let $0 \leq \tau$ be a finite stopping time. Then, by Itô's formula,

$$
\begin{aligned}
& \left|X^{n}(\tau)-X^{m}(\tau)\right|^{2} \\
& =2 \int_{0}^{\tau}\left\langle X^{n}(u)-X^{m}(u),\left(g\left(X_{u}^{n}+p_{u}^{n}\right)-g\left(X_{u}^{m}+p_{u}^{m}\right)\right) \mathrm{d} W(u)\right\rangle \\
& \quad+\int_{0}^{\tau} 2\left\langle f\left(X_{u}^{n}+p_{u}^{n}\right)-f\left(X_{u}^{m}+p_{u}^{m}\right), X^{n}(u)-X^{m}(u)\right\rangle \mathrm{d} u \\
& \quad+\int_{0}^{t}\|\| g\left(X_{u}^{n}+p_{u}^{n}\right)-g\left(X_{u}^{m}+p_{u}^{m}\right) \|^{2} \mathrm{~d} u .
\end{aligned}
$$


In order to use condition (M), note that by construction for $s>0$ and $s+u \leq 0$

$$
\bar{X}_{s}^{m}(u)=\bar{X}_{s}^{n}(u)=\varphi(s+u) .
$$

Hence, together with (3.3) and (3.4), the sum of the $\mathrm{d} u$-integrals on the r.h.s. can be estimated from above by

$$
\begin{aligned}
& \int_{0}^{\tau}\left(2\left\langle f\left(X_{u}^{n}+p_{u}^{n}\right)-f\left(X_{u}^{m}+p_{u}^{m}\right), p_{u}^{m}(0)-p_{u}^{n}(0)\right\rangle\right. \\
& \left.\quad+K_{R}\left\|X_{u}^{n}+p_{u}^{n}-\left(X_{u}^{m}+p_{u}^{m}\right)\right\|^{2}\right) \mathrm{d} u \\
& \leq \int_{0}^{\tau}\left(4 C_{1}(R)\left(\left|p_{u}^{n}(0)\right|+\left|p_{u}^{m}(0)\right|\right)\right. \\
& \left.\quad+4 K_{R}\left(\left\|p_{u}^{n}\right\|^{2}+\left\|p_{u}^{m}\right\|^{2}\right)+2 K_{R}\left\|X_{u}^{n}-X_{u}^{m}\right\|^{2}\right) \mathrm{d} u \\
& \leq \int_{0}^{\tau}\left[4 C_{1}(R)+4 K_{R} \widetilde{\rho}(R)\right]\left(\left\|p_{u}^{n}\right\|+\left\|p_{u}^{m}\right\|\right) \\
& \quad+2 K_{R} \sup _{v \in[0, u]}\left|X^{n}(v)-X^{m}(v)\right|^{2} \mathrm{~d} u
\end{aligned}
$$

provided $\tau \leq \tau_{R}^{m} \wedge \tau_{R}^{n} \wedge r_{R}=: \kappa$. Lemma 5.4 applies to $Z(s):=\mid X^{n}(s \wedge \kappa)-$ $\left.X^{m}(s \wedge \kappa)\right|^{2}$ with

$$
\begin{aligned}
& M(s):=2 \int_{0}^{s \wedge \kappa}\left\langle X^{n}(u)-X^{m}(u),\left(g\left(X_{u}^{n}+p_{u}^{n}\right)-g\left(X_{u}^{m}+p_{u}^{m}\right)\right) \mathrm{d} W(u)\right\rangle, \\
& H(s)=\int_{0}^{s \wedge \kappa}\left[4 C_{1}(R)+4 K(R) \widetilde{\rho}(R)\right]\left(\left\|p_{u}^{n}\right\|+\left\|p_{u}^{m}\right\|\right) \mathrm{d} u
\end{aligned}
$$

and $T=r_{R}$.

Once we have shown that some moment of $H^{*}(T):=\sup _{0 \leq s \leq T} H(s)$ converges to 0 as $n, m \rightarrow \infty$, Lemma 5.4 implies that for all $\varepsilon>0$,

$$
\lim _{m, n \rightarrow \infty} \mathbb{P}\left\{\sup _{s \in\left[0, \tau_{R}^{m} \wedge \tau_{R}^{n} \wedge r_{R}\right]}\left|X^{m}(s)-X^{n}(s)\right| \geq \varepsilon\right\}=0 .
$$

Since $H^{*}(T)$ is bounded uniformly in $\omega, n, m$, it suffices to show that $H^{*}(T)$ converges to zero in probability as $m, n \rightarrow \infty$ which can be verified as follows:

$$
p_{s}^{n}(u)= \begin{cases}0, & u \geq-r, u+s \leq \frac{\lfloor n s\rfloor}{n} \\ -\int_{\lfloor n s\rfloor / n}^{s+u} f\left(\bar{X}_{t}^{n}\right) \mathrm{d} t-\int_{\lfloor n s\rfloor / n}^{s+u} g\left(\bar{X}_{t}^{n}\right) \mathrm{d} W(t), & u+s \geq \frac{\lfloor n s\rfloor}{n}, u \leq 0\end{cases}
$$

implies

$$
\left\|p_{s}^{n}\right\| \leq \sup _{\lfloor n s\rfloor / n \leq t \leq s}\left|\int_{\lfloor n s\rfloor / n}^{t} f\left(\bar{X}_{u}^{n}\right) \mathrm{d} u\right|+\sup _{\lfloor n s\rfloor / n \leq t \leq s}\left|\int_{\lfloor n s\rfloor / n}^{t} g\left(\bar{X}_{u}^{n}\right) \mathrm{d} W(u)\right|,
$$


and hence - since $f$ and $g$ are bounded on $C_{R}-$

$\mathbb{E} \mathbf{1}_{\left\{\tau_{R}^{n} \geq s\right\}}\left\|p_{s}^{n}\right\| \rightarrow 0$ as $n \rightarrow \infty$, uniformly in $\left[0, r_{R}\right]$.

Therefore, $\mathbb{E} H^{*}(T)$ converges to 0 and (3.5) follows.

By definition of $\bar{X}^{m}$ this also yields

$$
\lim _{m, n \rightarrow \infty} \mathbb{P}\left\{\sup _{s \in\left[0, \tau_{R}^{m} \wedge \tau_{R}^{n} \wedge r_{R}\right]}\left\|\bar{X}_{s}^{m}-\bar{X}_{s}^{n}\right\| \geq \varepsilon\right\}=0 .
$$

Since $f, g$ are uniformly continuous on the compact set $C_{R}$,

$$
\lim _{m, n \rightarrow \infty} \mathbb{P}\left\{\sup _{s \in\left[0, \tau_{R}^{m} \wedge \tau_{R}^{n} \wedge r_{R}\right]}\left\{\left|f\left(\bar{X}_{s}^{m}\right)-f\left(\bar{X}_{s}^{n}\right)\right| \vee||\left|g\left(\bar{X}_{s}^{m}\right)-g\left(\bar{X}_{s}^{n}\right)\right|||\right\} \geq \varepsilon\right\}=0 .
$$

To further improve this statement, we apply Lemma 5.5 to

$$
\begin{aligned}
& X^{n}\left(s \wedge \tau_{R}^{m} \wedge \tau_{R}^{n} \wedge r_{R}\right)-X^{m}\left(s \wedge \tau_{R}^{m} \wedge \tau_{R}^{n} \wedge r_{R}\right) \\
& =\int_{0}^{s \wedge \tau_{R}^{m} \wedge \tau_{R}^{n} \wedge r_{R}}\left(F^{n}-F^{m}\right)(u) \mathrm{d} Z(u),
\end{aligned}
$$

where for simplicity we write

$$
Z(u)=(u, W(u)) \in \mathbb{R}^{m+1}, \quad F^{n}(u)=\left(f\left(\bar{X}_{u}^{n}\right), g\left(\bar{X}_{u}^{n}\right)\right) .
$$

Together with (3.7) this allows to conclude that for all $\varepsilon>0$

$$
\lim _{m, n \rightarrow \infty} \mathbb{P}\left\{\left\|X^{m}(\cdot)-X^{n}(\cdot)\right\|_{1 / 4 ;\left[0, \tau_{R}^{m} \wedge \tau_{R}^{n} \wedge r_{R}\right]} \geq \varepsilon\right\}=0 .
$$

Let us select a subsequence, which will again be denoted by $X^{n}$ such that

$$
\mathbb{P}\left\{\left\|X^{k}(.)-X^{l}(.)\right\|_{1 / 4 ;\left[0, \tau_{R}^{k} \wedge \tau_{R}^{l} \wedge r_{R}\right]} \geq 2^{-(l \wedge k)}\right\} \leq 2^{-(l \wedge k),}
$$

and define

$$
\tau_{R}=\liminf _{n \rightarrow \infty} \tau_{R}^{n}
$$

Due to (3.9), there is an $\left(\widetilde{F}_{t}\right)$-adapted process $X$ defined in $\left[0, \tau_{R}\right) \cap\left[0, r_{R}\right]$ to which $X^{n}$ converges $\mathbb{P}$-almost surely locally in $C^{1 / 4}\left(\left[0, \tau_{R}\right) \cap\left[0, r_{R}\right] ; \mathbb{R}^{d}\right)$. From (3.2), (3.5) and (3.7) and the continuity of $f$ and $g$ we infer that $X$ must be a solution to equation $(2.1)$ on $\left[0, \tau_{R}\right) \cap\left[0, r_{R}\right)$.

We remark that $\tau_{R}>0$ almost surely, which can be seen as follows. For any $\varepsilon>0$, using (3.9) we choose $n_{0}$ such that the set

$$
A=\left\{\omega \mid \sup _{k \geq n_{0}}\left\|X^{n_{0}}(\cdot)-X^{k}(\cdot)\right\|_{1 / 4 ;\left[0, \tau_{R}^{k} \wedge \tau_{R}^{n_{0}} \wedge r_{R}\right]}<\frac{R}{2}\right\}
$$


satisfies $\mathbb{P}(A) \geq 1-\varepsilon$. From $\bar{X}_{s}^{n_{0}}(\cdot)=\varphi((s+\cdot) \wedge 0) \in \Phi$ for $s \in\left[0, \frac{1}{n_{0}}\right]$, using Lemma 5.5 for the SDE (3.2) solved by $X^{n_{0}}$, it follows that

$$
\eta_{R / 2}^{n_{0}}:=\inf \left\{t \geq 0 \mid\left\|X^{n_{0}}(\cdot)-\varphi(0)\right\|_{1 / 4 ;[0, t]} \geq \frac{R}{2}\right\} \wedge r_{R}
$$

is strictly positive. By construction of $A$ it holds on $A$ that $\tau_{R}^{n} \wedge r_{R} \geq \eta_{R / 2}^{n_{0}} \wedge r_{R}$ for all $n \geq n_{0}$, hence in particular $\tau_{R}>0$.

Next, we show that almost surely one of the two following events occurs:

$$
\left\{\tau_{R} \geq r_{R}\right\} \quad \text { or } \quad\left\{\tau_{R}<r_{R}\right\} \cap\left\{\sup _{t<\tau_{R}}\|X(\cdot)-\varphi(0)\|_{1 / 4 ;[0, t]} \geq \frac{3 R}{4}\right\} .
$$

In case $\left\{\tau_{R} \geq r_{R}\right\}$, using (2.1) for $X(\cdot)$ on $\left[0, r_{R}\right)$ and the uniform boundedness of the coefficients on $C_{R}$ we may extend $X(\cdot)$ on the closed interval $\left[0, r_{R}\right]$ by setting

$$
X\left(r_{R}\right):=X(0)+\int_{0}^{r_{R}} f\left(X_{S}\right) \mathrm{d} s+\int_{0}^{r_{R}} g\left(X_{S}\right) \mathrm{d} W(s) .
$$

Together with (3.10) for

$$
\sigma_{R}:=\inf \left\{t \in\left[0, \tau_{R}\right) \cap\left[0, r_{R}\right] \mid\|X(\cdot)-\varphi(0)\|_{1 / 4 ;[0, t]} \geq \frac{R}{2}\right\} \wedge r_{R}
$$

this gives a well-defined process $t \mapsto X(t)$ for $t \in\left[0, \sigma_{R}\right]$ which solves (2.1) up to time $\sigma_{R}$ in the sense of Definition 2.1. Moreover, (3.1) holds by construction.

To prove (3.10) we show that the set

$$
B:=\left\{\tau_{R}<r_{R}\right\} \cap\left\{\sup _{t<\tau_{R}}\|X(\cdot)-\varphi(0)\|_{1 / 4 ;[0, t]}<\frac{3 R}{4}\right\} .
$$

has vanishing $\mathbb{P}$-measure. Assume the contrary, i.e. $\mathbb{P}(B)=p>0$. Then by (3.9) and the definition of $\tau_{R}$ we find some $n_{0} \in \mathbb{N}$ such that $\mathbb{P}(A)>\frac{p}{2}$, where

$$
A:=\left\{\begin{aligned}
& \sup _{k \geq n_{0}}\left\|X^{n_{0}}(\cdot)-X^{k}(\cdot)\right\|_{1 / 4 ;\left[0, \tau_{R}^{k} \wedge \tau_{R}^{n_{0}} \wedge r_{R}\right]}<\frac{R}{16} ; \\
& \inf _{n \geq n_{0}} \tau_{R}^{n}<r_{R} ; \quad \sup _{t<\tau_{R}}\|X(\cdot)-\varphi(0)\|_{1 / 4 ;[0, t]}<\frac{3 R}{4}
\end{aligned}\right\} .
$$

We show that in fact $\mathbb{P}(A)=0$. To this aim note that w.l.o.g. we may assume that $X^{n}$ converges to $X$ locally in $C^{1 / 4}\left(\left[0, \tau_{R}\right)\right)$ and

$$
\left[0, r_{R} \wedge \tau_{R}^{m}\right] \ni t \mapsto\left\|X^{m}(\cdot)\right\|_{1 / 4 ;[0, t]}
$$

is continuous for all $m \in \mathbb{N}$, for all $\omega \in A$, where the latter is again a consequence of Lemma 5.5. Now for $\omega \in A$ choose $m=m(\omega) \geq n_{0}$ such that $\tau_{R}^{m}<r_{R}$. Let

$$
\eta_{R}^{m}:=\inf \left\{t \geq 0 \mid\left\|X^{m}(\cdot)-\varphi(0)\right\|_{1 / 4 ;[0, t]} \geq R\right\} \leq \tau_{R}^{m},
$$


then by continuity $\eta_{7 R / 8}^{m}<\eta_{15 R / 16}^{m} \leq \tau_{R}^{n}$ for all $n \geq n_{0}$, hence $\eta_{7 R / 8}^{m}<\tau_{R}$. Again by continuity,

$$
\sup _{t<\eta_{7 R / 8}^{m}}\left\|X^{n}(\cdot)-\varphi(0)\right\|_{1 / 4 ;[0, t]} \geq \frac{3 R}{4}
$$

for all $n \geq n_{0}$ satisfying $\tau_{R}^{n}>\eta_{7 R / 8}^{m}$. In view of the convergence of $X^{n}$ to $X$ in $C^{1 / 4}\left[0, \eta_{7 R / 8}^{m}\right]$ for $n \rightarrow \infty$ this yields a contradiction to

$$
\sup _{t<\tau_{R}}\|X(\cdot)-\varphi(0)\|_{1 / 4 ;[0, t]}<\frac{3 R}{4} .
$$

Hence $A=\emptyset$ almost surely which proves (3.10).

To show uniqueness of a local solution, assume $X$ and $\tilde{X}$ are two solutions defined up to a stopping time $\tilde{\sigma} \leq \sigma_{R}$. Applying Itô's formula to the square of the norm of the difference of the solutions and using condition (M), Lemma 5.2 (with $C=0$ ) shows that the solutions agree on $[0, \tilde{\sigma}]$ almost surely.

Proof of Theorem 2.2. First we remark that it is sufficient to prove both the existence and uniqueness assertion of the theorem under the stronger assumption that $\mathbb{P}(\varphi \in \Phi)=1$ for any fixed compact subset $\Phi \subset \mathcal{C}$. In fact, since any probability measure on the Polish space $\mathcal{C}$ is tight, in both cases the general statement follows by approximation in $\mathbb{P}$-measure by initial conditions $\varphi_{n}=1_{\Phi_{n}}(\varphi) \cdot \varphi$, where e.g. the compact subsets $\Phi_{n} \subset \mathcal{C}$ are chosen such that $\mathbb{P}\left(\varphi \notin \Phi_{n}\right) \leq \frac{1}{n}$.

The proof of the existence statement is based on iterative use of Lemma 3.1. Recall for $R>0, r_{R}$ denotes the constant $r_{C}$ in condition (M) when $C=C_{\Phi, R}$. We may assume w.l.o.g. that the function $R \mapsto r_{R}$ is non-increasing and we may select a sequence $R^{(k)} \nearrow \infty, k \in \mathbb{N}$, such that $\sum_{k} r_{R^{(k)}}=\infty$.

Lemma 3.1 with $\Phi=: \Phi^{(1)}$ and $R:=R^{(1)}$ for initial condition $\varphi=: \varphi^{(1)} \in$ $\Phi^{(1)}$ guarantees the existence of a process $t \mapsto X(t)=: X^{(1)}(t), t \in\left[0, \sigma^{(1)}\right]$, with an $\mathscr{F}_{\text {. }}$-stopping time $\sigma^{(1)}:=\sigma_{R^{(1)}} \leq r_{R^{(1)}}$ which is a local solution to (2.1) on $\left[0, \sigma^{(1)}\right)$.

Next we may apply Lemma 3.1 to the same equation (2.1), now in the situation when $R$ and $W$ are chosen to be $R^{(2)}$ and $W_{t}^{(2)}=W\left(\sigma^{(1)}+t\right)-W\left(\sigma^{(1)}\right)$ on $(\Omega, \mathcal{F}, \mathbb{P})$ respectively, with $\mathcal{F}_{t}^{(2)}=\mathcal{F}_{t}{ }^{(2)} \vee \mathcal{N} \subset \mathcal{F}^{(2)}(t \geq 0)$, and $\mathcal{F}_{\text {. }}{ }^{(2)}$. independent initial condition $\varphi^{(2)}:=X_{\sigma^{(1)}}^{(1)} \in C_{\Phi, R_{1}}=: \Phi^{(2)}$. This yields an $\mathcal{F}^{(2)}$-stopping time $\tilde{\sigma}^{(2)} \leq r_{R^{(2)}}$ and a process $t \mapsto \tilde{X}^{(2)},\left[0, \tilde{\sigma}^{(2)}\right]$, solving (2.1) on $t \in\left[0, \tilde{\sigma}^{(2)}\right)$. (Note that here we have used the simple fact that $C_{C_{\Phi, R_{1}}, R_{2}}=$ 
$C_{\Phi, R_{2}}$ for $R_{2} \geq R_{1}$.) Hence, by continuation

$$
t \mapsto X^{(2)}(t)= \begin{cases}X^{(1)}(t) & \text { if } t \in\left[-r, \sigma^{(1)}\right] \\ \tilde{X}^{(2)}\left(t-\sigma^{(1)}\right) & \text { if } t \in\left(\sigma^{(1)}, \sigma^{(1)}+\tilde{\sigma}^{(2)}\right]\end{cases}
$$

we obtain an $\mathscr{F}$.-adapted process which is a local solution to equation (2.1) up to the $\mathcal{F}_{\text {.-stopping time }} \sigma^{(2)}=\sigma^{(1)}+\tilde{\sigma}^{(2)}$ in the sense of Definition 2.1 .

For general $n$ this construction is repeated inductively, furnishing a local solution $(X, \sigma)$ to equation (2.1) in the sense of Definition 2.1 where

$$
\sigma=\lim _{n \rightarrow \infty} \sigma^{(n)}
$$

To prove that $(X, \sigma)$ is maximal using the continuity of $f$ and $g$ it suffices to prove that the set

$$
\Sigma=\left\{\sup _{t \in[0, \sigma[}\left(\left|f\left(X_{t}\right)\right| \vee\left\|\left|g\left(X_{t}\right) \|\right|\right)<\infty\right\} \cap\{\sigma<\infty\}\right.
$$

has zero $\mathbb{P}$-measure. Now from the second statement in Lemma 3.1, from the construction of $X$ and from the property $\sum_{k} r_{R^{(k)}}=\infty$ it follows that

$$
\sup _{s \in[0, \sigma[}\left\|X(\cdot)-X\left(\sigma^{(k-1)}\right)\right\|_{1 / 4 ;[0, s]} \geq \frac{R^{(k)}}{2} \quad \mathbb{P} \text {-a.s. }
$$

for infinitely many $k \in \mathbb{N}$ on $\{\sigma<\infty\}$, i.e.

$$
\begin{array}{r}
\mathbb{P}(\Sigma)=\mathbb{P}\left(\sigma<\infty ; \sup _{s \in[0, \sigma)}\left(\left|f\left(X_{s}\right)\right| \vee\left\|\left|\left(X_{s}\right)\right|\right\|\right)<\infty ;\right. \\
\left.\sup _{s \in[0, \sigma)}\|X(\cdot)\|_{1 / 4 ;[0, s]}=\infty\right) .
\end{array}
$$

Since $X$ solves (2.1), due to e.g. Lemma 5.5, the r.h.s. is zero.

As for the uniqueness statement let $(Y, \tau)$ be another maximal solution with an associated sequence of announcing stopping times $\tau^{(n)}$. The construction of $X$ above yields a sequence of announcing stopping times $\sigma^{(n)}$ for $\sigma$ and compact sets $C_{n} \subset \mathcal{C}$ such that $X_{t \wedge \sigma^{(n)}} \in C_{n}$. Hence, by the same argument as in the proof

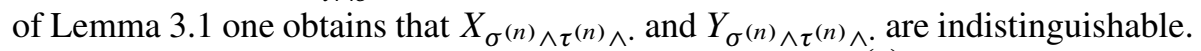
Moreover, the maximality of the pair $(Y, \tau)$ implies that $\sigma^{(n)}<\tau$ for all $n \in \mathbb{N}$, i.e. $\sigma \leq \tau$ almost surely. Conversely, the maximality of $\sigma$ implies $\sigma>\tau^{n}$, i.e. $\sigma \geq \tau$, which completes the proof. 


\section{Proof of Theorem 2.3}

Proof of Theorem 2.3. Let $(X, \sigma)$ be the maximal strong solution of equation (2.1). We want to show that $\sigma=\infty$ almost surely. Since $f$ and $g$ are bounded on bounded subsets of $\mathcal{C}$, it follows from (3.11) that $\lim _{\sup _{t} \lambda_{\sigma}}|X(t)|=\infty$ almost surely on the set $\{\sigma<\infty\}$. For a stopping time $0 \leq \tau<\sigma$, Itô's formula implies that

$$
\begin{aligned}
X^{2}(\tau)-X^{2}(0)= & \int_{0}^{\tau} 2\left\langle f\left(X_{u}\right), X(u)\right\rangle+\left\|\mid g\left(X_{u}\right)\right\|^{2} \mathrm{~d} u \\
& +2 \int_{0}^{\tau}\left\langle X(u), g\left(X_{u}\right) \mathrm{d} W(u)\right\rangle \\
\leq & \int_{0}^{\tau} \rho\left(\left\|X_{u}\right\|^{2}\right) \mathrm{d} u+M(\tau),
\end{aligned}
$$

where $M$ is a continuous local martingale. Applying Lemma 5.1 to $Z(t):=X^{2}(t)$ finishes the proof.

\section{Appendix}

We start by proving three lemmas which could be called stochastic Gronwall lemmas. We use them in the proof of Theorems 2.2 and 2.3. Then we prove a result about the tails of Hölder norms of stochastic integrals which we owe to Steffen Dereich (TU Berlin). We believe that all these results are of independent interest. In all lemmas, we assume that a filtered probability space $\left(\Omega, \mathcal{F}_{,}\left(\mathcal{F}_{t}\right)_{t \geq 0}, \mathbb{P}\right)$ is given and that it satisfies the usual conditions. Throughout, we will use the notation $Z^{*}(T)=\sup _{0 \leq t \leq T} Z(t)$ for a real-valued process $Z$.

Lemma 5.1. Let $\sigma>0$ be a stopping time and let $Z$ be an adapted non-negative stochastic process with continuous paths defined on $[0, \sigma)$ which satisfies the inequality

$$
Z(t) \leq \int_{0}^{t} \rho\left(Z^{*}(u)\right) \mathrm{d} u+M(t)+C,
$$

and $\lim _{t \uparrow \sigma} Z^{*}(t)=\infty$ on $\{\sigma<\infty\}$ almost surely. Here, $C \geq 0$ and $M$ is a continuous local martingale defined on $[0, \sigma), M(0)=0$ and $\rho:[0, \infty) \rightarrow(0, \infty)$ is non-decreasing, and $\int_{0}^{\infty} 1 / \rho(u) \mathrm{d} u=\infty$. Then $\sigma=\infty$ almost surely. 
Proof. Let $Y$ be the unique (maximal) solution of the equation

$$
Y(t)=\int_{0}^{t} \rho\left(Y^{*}(u)\right) \mathrm{d} u+M(t)+C .
$$

Clearly, $Y(t) \geq Z(t)$ for all $t$ for which $Y$ is defined and therefore it suffices to prove the claim for $Y$ instead of $Z$. For $a>C$, define $\tau_{a}:=\inf \{t \geq 0 \mid Y(t) \geq a\}$. For $C<a<b$ and $\delta>0$ we get

$$
\mathbb{P}\left\{\tau_{b}-\tau_{a} \leq \delta \mid \mathcal{F}_{\tau_{a}}\right\} \leq \mathbb{P}\left\{b-a \leq \delta \rho(b)+\sup _{t \in\left[\tau_{a}, \tau_{b} \wedge\left(\tau_{a}+\delta\right)\right]} M(t)-M\left(\tau_{a}\right) \mid \mathcal{F}_{\tau_{a}}\right\}
$$

on the set $\left\{\tau_{a}<\infty\right\}$. Note that on $\left\{\tau_{a}<\infty\right\}$ we have

$$
M(t)-M\left(\tau_{a}\right) \geq Y(t)-Y\left(\tau_{a}\right)-\left(t-\tau_{a}\right) \rho(b) \geq-a-\delta \rho(b)
$$

for $\tau_{a} \leq t \leq \tau_{b} \wedge\left(\tau_{a}+\delta\right)$ since $Y$ is non-negative. For

$$
\tau:=\inf \left\{t \geq \tau_{a} \mid M(t)-M\left(\tau_{a}\right) \geq b-a-\delta \rho(b)\right\} \wedge \tau_{b} \wedge\left(\tau_{a}+\delta\right)
$$

we therefore get

$$
0=\mathbb{E}\left(M(\tau)-M\left(\tau_{a}\right) \mid \mathcal{F}_{\tau_{a}}\right) \geq(b-a-\delta \rho(b)) p-(a+\delta \rho(b))(1-p),
$$

where $p:=\mathbb{P}\left\{M(\tau)-M\left(\tau_{a}\right) \geq b-a-\delta \rho(b) \mid \mathcal{F}_{\tau_{a}}\right\}$. Hence

$$
\mathbb{P}\left\{\tau_{b}-\tau_{a} \leq \delta \mid \mathcal{F}_{\tau_{a}}\right\} \leq p \leq \frac{a+\delta \rho(b)}{b} \quad \text { on }\left\{\tau_{a}<\infty\right\} .
$$

Fix $a>C$. Then

$$
\sigma=\tau_{a}+\sum_{k=1}^{\infty}\left(\tau_{2^{k} a}-\tau_{2^{k-1} a}\right) .
$$

We show that the sum diverges almost surely. To ease notation, we write $\tau_{k}$ instead of $\tau_{2^{k} a}$. For $\delta_{k}>0, k \in \mathbb{N},(5.2)$ implies that

$$
\mathbb{P}\left\{\tau_{k}-\tau_{k-1} \geq \delta_{k} \mid \mathcal{F}_{\tau_{k-1}}\right\} \geq \frac{1}{2}-\delta_{k} \frac{\rho\left(2^{k} a\right)}{2^{k} a}
$$

on the set $\left\{\tau_{k-1}<\infty\right\}$. Now

$$
\sigma \geq \sum_{k=1}^{\infty}\left(\tau_{k}-\tau_{k-1}\right) \geq \sum_{k=1}^{\infty} \delta_{k} 1_{\left\{\tau_{k}-\tau_{k-1} \geq \delta_{k}\right\}} .
$$


We choose

$$
\delta_{k}:=\frac{1}{4} \frac{2^{k} a}{\rho\left(2^{k} a\right)}, \quad k \in \mathbb{N} .
$$

Since $\rho$ is non-decreasing we have

$$
\sum_{k=1}^{\infty} \delta_{k} \geq \frac{1}{4} \int_{2 a}^{\infty} \frac{1}{\rho(u)} \mathrm{d} u=\infty
$$

and

$$
\mathbb{P}\left\{\tau_{k}-\tau_{k-1} \geq \delta_{k} \mid \mathcal{F}_{\tau_{k-1}}\right\} \geq \frac{1}{4} \text { on }\left\{\tau_{k-1}<\infty\right\} .
$$

It follows (e.g. from Kolmogorov's three series theorem) that the right hand side of (5.3) diverges on the set $\left\{\tau_{k}<\infty\right.$ for all $\left.k \in \mathbb{N}\right\}$. On the complement of this set, $\sigma$ is also infinite, i.e. the proof of the lemma is complete.

While the previous lemma was concerned with non-blow up of $Z$, the following lemma shows that $Z$ remains small in case the initial condition is small. In principle we could formulate the following lemma also using a function $\rho$ as in the previous one but we prefer not to in order to obtain a reasonably explicit formula for moments of $Z^{*}(T)$.

Lemma 5.2. Let $Z$ be an adapted non-negative stochastic process with continuous paths defined on $[0, \infty)$ which satisfies the inequality

$$
Z(t) \leq K \int_{0}^{t} Z^{*}(u) \mathrm{d} u+M(t)+C,
$$

where $C \geq 0, K>0$ and $M$ is a continuous local martingale with $M(0)=0$.

Then for each $0<p<1$, there exist universal finite constants $c_{1}(p), c_{2}(p)$ (not depending on $K, C, T$ and $M$ ) such that

$$
\mathbb{E}\left(Z^{*}(T)\right)^{p} \leq C^{p} c_{2}(p) \exp \left\{c_{1}(p) K T\right\} \quad \text { for every } T \geq 0 .
$$

Proof. Let $Y$ be the unique solution of the equation

$$
Y(t)=K \int_{0}^{t} Y^{*}(u) \mathrm{d} u+M(t)+C .
$$

Clearly, $Y(t) \geq Z(t)$ for all $t \geq 0$ and therefore it suffices to prove the claim for $Y$ instead of $Z$. Let $\tau_{a}:=\inf \{t \geq 0: Y(t) \geq a\}$. Like in the proof of Lemma 5.1, we obtain for $\beta \in(0,1)$ and $b>a \geq C$

$$
\mathbb{P}\left\{\tau_{b}-\tau_{a} \leq \frac{\beta}{K} \mid \mathcal{F}_{\tau_{a}}\right\} \leq \frac{a+\beta b}{b} \text { on }\left\{\tau_{a}<\infty\right\} .
$$


For $T>0, m \in \mathbb{N}, \gamma>(1-\beta)^{-1}$ we get

$$
\mathbb{P}\left\{Y^{*}(T) \geq \gamma^{m} C\right\}=\mathbb{P}\left\{\tau_{\gamma^{m} C} \leq T\right\}=\mathbb{P}\left\{\sum_{i=1}^{m} \tau_{\gamma^{i} C}-\tau_{\gamma^{i-1} C} \leq T\right\} .
$$

By (5.4), the last sum is stochastically larger than $\beta / K$ times a binomial variable $V$ with parameters $m$ and $\alpha:=1-\frac{1}{\gamma}-\beta$. Therefore, for $\lambda>0$ and $N:=\left\lceil\frac{K T}{\beta}\right\rceil$ we get

$$
\mathbb{P}\left\{Y^{*}(T) \geq \gamma^{m} C\right\} \leq \mathbb{P}\{V \leq N\}=\mathbb{P}\left\{\mathrm{e}^{-\lambda V} \geq \mathrm{e}^{-\lambda N}\right\} .
$$

Applying Markov's inequality, representing $V$ as a sum of $m$ independent Bernoulli $(\alpha)$ variables and optimizing over $\lambda>0$ as usual, we obtain for $m \geq$ $\left\lceil\frac{N}{\alpha}\right\rceil=: m_{0}$

$$
\begin{gathered}
\mathbb{P}\left\{Y^{*}(T) \geq \gamma^{m} C\right\} \leq \exp \left\{(m-N) \log \frac{m}{m-N}+(m-N) \log (1-\alpha)\right. \\
\left.+N \log \alpha+N \log \frac{m}{N}\right\} .
\end{gathered}
$$

Assume that $p \log \gamma+\log (1-\alpha)<0$ (which requires $p<1$ since $1-\alpha=$ $\left.\frac{1}{\gamma}+\beta>\frac{1}{\gamma}\right)$ and fix $q>0$ such that $p \log \gamma+\log (1-\alpha)+q^{-1}<0$. Then

$$
\begin{aligned}
& \mathbb{E} Y^{*}(T)^{p}= \int_{0}^{\infty} \mathbb{P}\left\{Y^{*}(T) \geq s^{1 / p}\right\} \mathrm{d} s \\
& \leq \gamma^{m_{0} p} C^{p}+\sum_{m=m_{0}}^{\infty} C^{p} \gamma^{p m}(\gamma-1) \exp \left\{(m-N) \log \frac{m}{m-N}\right. \\
&\left.\quad+(m-N) \log (1-\alpha)+N \log \alpha+N \log \frac{m}{N}\right\} \\
& \leq \gamma^{m_{0} p} C^{p}+C^{p}(\gamma-1) \exp \left\{N \log \frac{\alpha q}{1-\alpha}\right\} \\
& \quad \times \sum_{m=m_{0}}^{\infty} \exp \left\{m\left(p \log \gamma+\log (1-\alpha)+q^{-1}\right)\right\} \\
&= C^{p}\left(\gamma^{m_{0} p}+(\gamma-1) \exp \left\{N \log \frac{\alpha q}{1-\alpha}\right\}\right. \\
&\left.\quad \times \frac{\exp \left\{m_{0}\left(p \log \gamma+\log (1-\alpha)+q^{-1}\right)\right\}}{1-\exp \left\{p \log \gamma+\log (1-\alpha)+q^{-1}\right\}}\right),
\end{aligned}
$$

where we used the inequalities

$$
\log (1+x) \leq x \quad\left(\text { for } x=\frac{N}{m-N}\right)
$$

and

$$
\log x \leq \log q+q^{-1}(x-q) \quad\left(\text { for } x=\frac{m}{N}\right)
$$


in the last " $\leq$ ". Observing that

$$
m_{0} \leq\left(\frac{k T}{\beta}+1\right) \frac{1}{\alpha}+1 \quad \text { and } \quad N \leq \frac{K T}{\beta}+1,
$$

the claim follows.

Remark 5.3. It is clear that the previous lemma does not hold for $p>1$ : just consider a scalar geometric Brownian motion starting with $C$. Its $p^{\text {th }}$ moment for $p>1$ at time 1 (say) is unbounded with respect to the volatility $\sigma$. We don't know whether the lemma holds true for $p=1$ but we conjecture that it doesn't.

Lemma 5.4. Let $Z$ be an adapted non-negative stochastic process with continuous paths defined on $[0, \infty)$ which satisfies the inequality

$$
Z(t) \leq K \int_{0}^{t} Z^{*}(u) \mathrm{d} u+M(t)+H(t),
$$

where $K>0, M$ is a continuous local martingale with $M(0)=0$, and $H$ is an adapted process with continuous paths satisfying $H(0)=0$. Then, for each $0<p<1$ and $\alpha>\frac{1+p}{1-p}$, there exist constants $c_{3}, c_{4}$ depending on $p, \alpha$ only such that

$$
\mathbb{E}\left(Z^{*}(T)\right)^{p} \leq c_{3} \exp \left\{c_{4} K T\right\}\left(\mathbb{E} H^{*}(T)^{\alpha}\right)^{p / \alpha} \quad \text { for every } T \geq 0 .
$$

Proof. Fix $T>0$ and for $i \in \mathbb{N}$ let $X_{i}$ be the unique solution of

$$
X_{i}(t)=K \int_{0}^{t} X_{i}^{*}(u) \mathrm{d} u+M(t)+i .
$$

Hence, $Z \leq X_{i}$ on $[0, T] \times \Omega_{i}$ where

$$
\Omega_{i}:=\left\{\omega: \sup _{0 \leq t \leq T} H(t) \leq i\right\} .
$$

Let $s \in\left(\frac{1}{1-p}, \frac{\alpha}{1+p}\right)$ and let $r>1$ be defined by $r^{-1}+s^{-1}=1$. Then $p r<1$ and Lemma 5.2 and Hölder's inequality imply

$$
\begin{aligned}
\mathbb{E}\left(Z^{*}(T)\right)^{p} & \leq \sum_{i=1}^{\infty} \mathbb{E}\left(\left(X_{i}^{*}(T)\right)^{p_{1}}{ }_{\Omega_{i} \backslash \Omega_{i-1}}\right) \\
& \leq \sum_{i=1}^{\infty}\left(\mathbb{E}\left(X_{i}^{*}(T)\right)^{p r}\right)^{1 / r} \mathbb{P}\left\{\Omega_{i} \backslash \Omega_{i-1}\right\}^{1 / s}
\end{aligned}
$$




$$
\begin{aligned}
\leq & \sum_{i=1}^{\infty} i^{p} c_{2}(p r)^{1 / r} \exp \left\{K T c_{1}(p r) / r\right\} \mathbb{P}\left\{H^{*}(T) \geq i-1\right\}^{1 / s} \\
\leq & \exp \left\{K T c_{1}(p r) / r\right\} c_{2}(p r)^{1 / r} \\
& \times\left(\left(\mathbb{E} H^{*}(T)^{\alpha}\right)^{1 / s} \sum_{i=2}^{\infty} i^{p}(i-1)^{-\alpha / s}+1\right),
\end{aligned}
$$

where we used Markov's inequality in the last step.

For each $\xi>0$, the inequality in the assumption of the lemma remains true if $H, M$, and $Z$ are multiplied by $\xi$. Therefore, the inequality

$$
\begin{aligned}
\mathbb{E}\left(Z^{*}(T)\right)^{p} \leq & \exp \left\{K T c_{1}(p r) / r\right\} c_{2}(p r)^{1 / r} \\
& \times\left(\xi^{\frac{\alpha}{s}-p}\left(\mathbb{E} H^{*}(T)^{\alpha}\right)^{1 / s} \sum_{i=2}^{\infty} i^{p}(i-1)^{-\alpha / s}+\xi^{-p}\right)
\end{aligned}
$$

follows. Optimizing the right hand side over $\xi>0$ yields the assertion of the lemma.

Lemma 5.5 (S. Dereich). For $m, d \in \mathbb{N}, \alpha \in\left(0, \frac{1}{2}\right)$ and $t_{0}>0$ there exist some universal strictly positive constants $c_{i}=c_{i}\left(d, m, \alpha, t_{0}\right), i=1,2,3$, such that for $Z(t)=(t, W(t)) \in \mathbb{R}^{m+1}$ with an $\mathbb{R}^{m}$-valued Brownian motion $W$

$$
\mathbb{P}\left(\left\|\int_{\sigma}^{(\cdot)} F \mathrm{~d} Z\right\|_{\alpha ;[\sigma, \tau]} \geq u\right) \leq c_{1} e^{-c_{2} u^{2} / v^{2} T}
$$

for

$$
\frac{u}{v\left(T+T^{1-\alpha}\right)} \geq c_{3}, \quad T \geq t_{0}
$$

for any pair $\sigma \leq \tau$ of finite $\left(\mathscr{F}_{t}\right)$-stopping times with $\tau-\sigma \leq T$ and any $\left(\mathscr{F}_{t}\right)$ -

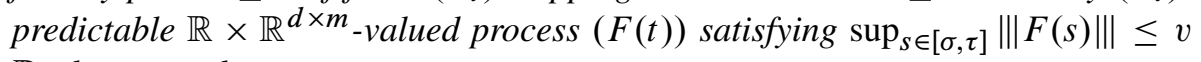
$\mathbb{P}$-almost surely.

Proof. It suffices to treat the case when $\sigma=0$ and $m=d=1$, where we have to deal with real-valued semimartingales of the form

$$
t \mapsto \int_{0}^{t} F(s) \mathrm{d} s=: A(t) \quad \text { or } \quad t \mapsto \int_{0}^{t} F(s) \mathrm{d} W(s)=: M(t)
$$

with integrands satisfying $\sup _{s \in[0, T]}|F(s)| \leq v$ almost surely. The first case is easy: the map $t \mapsto A(t)$ is Lipschitz with constant (at most) $v$ and therefore $\|A(\cdot)\|_{\alpha ;[0, \tau]} \leq v\left(T+T^{1-\alpha}\right)$ almost surely, so the claim follows in this case. Let 
us consider $M$. The Gaussian isoperimetric inequality, cf. e.g. [2, Section 4.3], implies the existence of some universal positive constants $k_{i}=k_{i}(\alpha), i=1,2$ such that

$$
\mathbb{P}\left(\|W(\cdot)\|_{\alpha ;[0,1]} \geq u\right) \leq k_{1} e^{-k_{2} u^{2}} \quad \text { for } u \geq 0 .
$$

We choose an independent Brownian motion $W^{\prime}$ and let

$$
F^{\prime}(s)=\sqrt{v^{2}-F^{2}(s)} .
$$

Then both processes

$$
t \mapsto B^{(j)}(t)=\int_{0}^{t} F(s) \mathrm{d} W(s)-(-1)^{j} \int_{0}^{t} F^{\prime}(s) \mathrm{d} W^{\prime}(s), \quad j=1,2,
$$

have the same distribution as $t \mapsto v W(t)$. From

$$
B^{(1)}(t)+B^{(2)}(t)=2 \int_{0}^{t} F(s) \mathrm{d} W(s)
$$

and the triangle inequality in $C^{\alpha}$ one gets

$$
\begin{aligned}
\mathbb{P}\left(\left\|\int_{0}^{(\cdot)} F(s) \mathrm{d} W(s)\right\|_{\alpha ;[0, \tau]} \geq u\right) & \leq 2 \mathbb{P}\left(\|v W(\cdot)\|_{\alpha ;[0, T]} \geq u\right) \\
& \leq 2 \mathbb{P}\left(\|W(\cdot)\|_{\alpha ;[0,1]} \geq \frac{u}{v \sqrt{T}\left(T^{-\alpha} \vee 1\right)}\right) \\
& \leq 2 k_{1} \exp \left\{-k_{2} \frac{u^{2}}{v^{2} T\left(T^{-\alpha} \vee 1\right)^{2}}\right\},
\end{aligned}
$$

which yields the claim of the lemma.

Remark. Alternatively, the previous lemma can be proved using the fact that each continuous local martingale starting at 0 can be represented as a time-changed Brownian motion.

Acknowledgments. This work was partially supported by the DFG-Forschergruppe 718 "Analysis and Stochastics in Complex Physical Systems".

\section{Bibliography}

[1] L. A. Alyushina, Euler polygonal lines for Itô equations with monotone coefficients, Teor. Veroyatnost. i Primenen. 32 (1987), 367-373.

[2] V. I. Bogachev, Gaussian Measures, American Mathematical Society, Providence, R.I., 1998. 
[3] E. Buckwar, R. Kuske, S.-E. Mohammed and T. Shardlow, Weak convergence of the Euler scheme for stochastic differential delay equations, LMS J. Comput. Math. 11 (2008), 60-99.

[4] E. Clément, A. Kohatsu-Higa and D. Lamberton, A duality approach for the weak approximation of stochastic differential equations, Ann. Appl. Probab. 16 (2006), 1124-1154.

[5] P. E. Kloeden and A. Neuenkirch, The pathwise convergence of approximation schemes for stochastic differential equations, LMS J. Comput. Math. 10 (2007), 235253.

[6] N. V. Krylov, A simple proof of the existence of a solution to the Ito equation with monotone coefficients, Teor. Veroyatnost. i Primenen. 35 (1990), 576-580.

[7] N. V. Krylov, On Kolmogorov's equations for finite-dimensional diffusions, in: Stochastic PDE's and Kolmogorov Equations in Infinite Dimensions (Cetraro, 1998), Lecture Notes in Mathematics 1715, pp. 1-63, Springer, Berlin, 1999.

[8] U. Küchler and E. Platen, Strong discrete time approximation of stochastic differential equations with time delay, Math. Comput. Simulation 54 (2000), 189-205.

[9] X. Mao Stochastic Differential Equations and Applications, Horwood, Chichester, 1997.

[10] S. E. A. Mohammed, Stochastic Functional Differential Equations, Pitman, Boston, 1984.

[11] C. Prévôt and M. Röckner, A Concise Course on Stochastic Partial Differential Equations, Lecture Notes in Mathematics 1905, Springer, Berlin, 2007.

[12] D. Xu, Z. Yang and Y. Huang, Existence-uniqueness and continuation theorems for stochastic functional differential equations, J. Differential Equations 245 (2008), 1681-1703.

Received June 1, 2009; accepted November 4, 2009.

\section{Author information}

Max-K. von Renesse, Institute of Mathematics, Technische Universität Berlin, Germany. E-mail: mrenesse@math.tu-berlin.de

Michael Scheutzow, Institute of Mathematics, Technische Universität Berlin, Germany. E-mail: ms@math.tu-berlin.de 